\title{
Academicians' Burnout, Vigor and Occupational Satisfactions in the Department of Fine Arts Education: A Sample of Uludag University
}

\author{
Rasim Erol Demirbatır ${ }^{1}$, Doruk Engür ${ }^{1}$ \\ ${ }^{1}$ Uludag University, Turkey \\ Correspondence: Doruk Engür, Uludag University, Turkey.
}

Received: April 19, 2018

Accepted: May 2, 2018

Online Published: June 19, 2018

doi:10.11114/jets.v6i7.3219

URL: https://doi.org/10.11114/jets.v6i7.3219

\begin{abstract}
The success of an educational institution is heavily based on instructors' occupational satisfaction and their happiness at their workplace. Therefore, determining the case of the instructors' wellbeing in order to improve working standards is really important to sustain high quality in education. In this sense, this study set out to identify state of academicians in terms of their level of burnout, vigor and occupational satisfaction at a department of Fine Arts Education at Uludag University. All 42 academician participants attended the study. To determine the level of burnout and vigor, Shirom-Melamed Burnout Measure was employed, and to learn participants' occupational satisfaction levels, a questionnaire developed by authors was used. The results show that burnout and vigor have negative correlation and occupational satisfaction has negatively correlated with emotional exhaustion, which is a sub-dimension of burnout but positively correlated with physical strength, which is a sub-dimension of vigor. Moreover, it is determined that the academicians who go to the doctor less frequently have higher occupational satisfaction and the academicians who are facing health issues have higher level of burnout. The results confirm that health status significantly affects one sub-dimension of burnout, which is physical fatigue.
\end{abstract}

Keywords: burnout, vigor, occupational satisfaction, fine arts education

\section{Introduction}

Occupational satisfaction is the most common issue of today's modern world. People working in almost all professions work longer and more, which decreases occupational satisfactions and increases their level of burnout. Occupational satisfaction can be defined as a body of feelings that a person has towards inner and outer factors related with his job (Kilic, Tanrikulu and Ugur, 2013). Furthermore, high burnout level is associated to unwell job satisfaction (Evans et al., 2006; Gursel and Sunbul, 2002; Koustelios and Tsigilis, 2005; Ogresta, Rusac and Zorec, 2008; Ozyurt, Hayran and Sur, 2006) and job burnout is also common as a result of intense relations and stressful workplaces (Filiz, 2014). Burnout was first defined by Freudenberger in 1974 as the feeling physically and emotionally exhausted due to the job conditions. Unfortunately, when compared to other professions, teachers have high levels of exhaustion (Maslach, Jackson, and Leiter, 1996; Schaufeli and Enzmann, 1998).

In academia, the level of burnout and satisfaction affects the quality of the education, the number of the contributions into the field of science and bringing innovations to their own field. In other words, only when the academic staff is satisfied with their working conditions, they can become creative, productive and ready to create new ideas for their profession. Karabiyik, Eker and Anbar (2008)'s study determines that work environment, administrative workload, academic workload, promotion and evaluation and research fund have effect on burnout among academicians. It is known that burnout is a serious issue that could lead many teachers to resign (Cooper, 2016). Maslach, Schaufeli and Leiter (2001) divide job burnout into three dimensions which are exhaustion, cynicism and inefficiency. Burnout was initially found in professions that involve interaction with people (Bayram, Gursakal, and Bilgel, 2010). This is probably because of the intensity of interpersonal relations when they take up too much of the job. It is known that a bullying management style, often faced by professionals such as healthcare workers and teachers, has a damage on personal health, morale and performance (Cooper, 2016).

Academic vigor, on the other hand, refers to having sufficient amount of energy and mental wellbeing while engaging academic work; having enthusiasm and inspiration towards their job; and being able to focus on work, as well as feeling happy (Schaufeli, Salanova, Gonzáles-Romá and Bakker, 2002; Schaufeli and Bakker, 2004). Derinbay (2012) finds negative 
correlation between occupational satisfaction and burnout, which means the occupational satisfaction increases as the burnout level decreases. It may not be wrong to conclude that the academicians whose level of vigor is high find the urge to take their teaching one step ahead and expand their horizon and viewpoints so as to be better educators in their field.

In a study, which is carried out in Ondokuz Mayis University, it is found that female academicians have significantly higher level of burnout when compared to male academicians (Cavusoglu, Unver, Dogan, Islamoglu, and Ozdemir, 2015). Derinbay (2012) who studies the burnout levels of academicians using Maslach Burnout Inventory states that emotional exhaustion (a sub-dimension of Burnout) is higher among female academicians and also occupational satisfaction is higher among male academicians. However, there is no significant difference in either other sub-dimensions (depersonalization and personal success levels) or total burnout scores, when analyzed in terms of gender. Kucuksuleymanoglu (2007), in her study carried out in the Department of Education at Uludag University, states that female academicians, single academicians and academicians age between 21 and 30 have higher level of burnout. Otacioglu (2008) carries out a study on in-service music teachers and finds out that male teachers and novice teachers' level of burnout is higher and teachers in state schools feel burnout more than the ones working in private schools.

Unfortunately, the literature on the burnout and vigor levels of academicians at Fine Arts Education Departments is not enough to obtain accurate information to determine the state of academicians' levels of burnout and vigor. Therefore, this current study set out to determine the burnout, vigor and occupational satisfaction levels of academicians who work at the Department of Fine Arts Education.

\section{Method}

\subsection{Research Model}

This study is an associational research. Fraenkel, Wallen, and Hyun (2012) describe the associational research as a research in which the relationships among variables are studied without manipulating them. In this study, firstly, participants' health status and the number of doctor visits in a year were analyzed in terms of gender and age. Following this, academicians' burnout, vigor and occupational satisfaction levels were analyzed in terms of gender, age, health status, doctor visit in a year, and years of service. The correlations between Burnout, vigor and occupational satisfaction levels were also investigated.

\subsection{Sampling}

Forty-two academic staff working in Fine Arts Education Department participated voluntarily to the study. 24 participants were female (mean age $=42.21, S D=8.85$ ) and 18 participants were male (mean age $=49.61, S D=9.15$ ). The average years of service for participants is $20.80(S D=9.11)$. Table 1 shows detailed information about academicians participated in the study. Data about variables age, health status, number of doctor visits in a year and years of service were collected in a continuous scale. In order for readers to understand easier, table also shows grouped frequencies.

Table 1. Demographic characteristics of the participants

\begin{tabular}{lllll}
\hline & $\mathrm{N}$ & $\%$ & $M \pm S D$ & $M d n$ \\
\hline Gender & & & & \\
Male & 18 & 42.9 & & \\
Female & 24 & 57.1 & & \\
Age & 5 & 11.9 & $45.38 \pm 9.61$ & 44.00 \\
$28-32$ & 4 & 9.5 & & \\
$33-37$ & 4 & 9.5 & & \\
$38-42$ & 15 & 35.7 & & \\
$43-47$ & 6 & 14.3 & & \\
$48-52$ & 3 & 7.1 & & \\
$53-57$ & 5 & 11.9 & & \\
$58+$ & 2 & 4.8 & $7.19 \pm 1.22$ & 7.00 \\
Health Status & 13 & 31.0 & & \\
5,00 & 10 & 23.8 & & \\
6,00 & 9 & 21.4 & & \\
7,00 & 8 & 19.0 & & \\
8,00 & & & $3.33 \pm 3.91$ & 2.00 \\
9,00 & 23 & 54.8 & & \\
Number of doctor visits in a year & 12 & 28.6 & & \\
$0-2$ & 4 & 9.5 & & \\
$3-5$ & 3 & 7.1 & & \\
$6-8$ & 6 & 14.3 & $20.81 \pm 9.11$ & 21.00 \\
$9+$ & 10 & 23.8 & & \\
Years of Service & 16 & 38.1 & & \\
$15-11$ & 5 & 11.9 & & \\
$12-18$ & 5 & 11.9 & & \\
$19-25$ & & & \\
$26-32$ & $33+$ & & & \\
\hline
\end{tabular}

*For the evaluation of the health status of the participants a self-reporting 10-point scale was used (1 very bad to 10 excellent) 


\subsection{Data Collection Tools}

In the study, participants' burnout levels were measured via Shirom-Melamed Burnout Measure (2005). Translated into Turkish by Bilgel, Bayram, Ozdemir, Dogan and Ekin (2012), Shirom-Melamed Burnout Measure (SMBM) has three subscales. The three subscales are the physical fatigue (PF) factor (6 items), the cognitive weariness $(\mathrm{CW})$ factor $(5$ items), and the emotional exhaustion (EEx) factor (3 items). All 14 items were measured on a 7-point Likert-type scale. Bilgel et al. (2012) confirmed the three-factor structure of the Turkish version of Shirom-Melamed Burnout Measure. In the recent study The Cronbach $\alpha$ calculated to assess the internal consistency for total scale is 0.91 ; for PF factor it is 0.93; for $\mathrm{CW}$ factor it is 0.89 and for EEx factor it is 0.86 .

In order to measure vigor levels Shirom-Melamed Vigor Measure (2005) was employed. The Shirom-Melamed Burnout Measure (SMVM) was translated into Turkish and its three-factor structure was confirmed by Bilgel et al. (2012). Three factors are physical strength (PHY, 5 items); emotional energy (EE, 4 items); and cognitive liveliness (CL, 3 items). All 14 items were measured on a 7-point Likert-type scale. In the recent study the Cronbach $\alpha$ calculated to assess the internal consistency for total scale is 0.88 ; for PHY factor it is 0.87 ; for EE factor it is 0.86 and for CL factor it is 0.84 .

Participants' occupational satisfaction levels were measured by a questionnaire (OSQ) developed by authors (see appendix). In the process of writing the items, a semi-structured interview with 10 academic staff was conducted. They were asked to tell what would make them satisfy with their occupations. After the interview 20 statements that show the occupational satisfaction were chosen for the questionnaire. These 20 items were measured using a 5-point Likert-type scale, where $1=$ strongly disagree, and $5=$ strongly agree. The Cronbach $\alpha$ calculated to assess the internal consistency of OSQ is 0.85

In order to determine participants' feeling about their health, participants were asked to indicate their health condition using a 10-point scale, from $1=$ very unhealthy to $10=$ very healthy.

\subsection{Data Analysis}

In order to analyze participants' health status and the number of doctor visits in a year in terms of gender and age Mann-Whitney U test was employed. Burnout, vigor and occupational satisfaction levels were analyzed in terms of gender, age, health status, the number of doctor visits in a year, and years of service using t-tests. Pearson correlation coefficients were calculated in order to determine the relationship between burnout, vigor and occupational satisfaction levels.

\section{Results}

\subsection{Analysis of Health Scores and the Number of Doctor Visits by Gender and Age}

The mean health scores of the participants were $7.19 \pm 1.21$ (women $=7.20 \pm 1.28$, men $=7.16 \pm 1.15$ ) No statistically significant difference was found between the mean health scores of male and female participants, $U=209.5, p=0.865$. The mean health scores of the participants who were 44 years old and younger were $7.09 \pm 1.10$; the mean health scores of those aged over 44 were $7.30 \pm 1.34$. There was no statistically significant difference between health scores in terms of age, $U=202, p=0.640$.

The average number of doctor visits was $3.33 \pm 3.90$ (women $=3.54 \pm 4.26$, men $=3.05 \pm 3.47$ ). There was no statistically significant difference between the number of doctor visits of women and men, $U=204.5 p=0.767$. The average number of doctor visits of the participants who were 44 years old and younger was $3.31 \pm 3.53$; for those over 44 , it was $3.35 \pm 4.36$. There was no statistically significant difference in the number of doctor visits in terms of age, $U$ $=2016, p=0.919$.

\subsection{Analysis of SMBM, SMVM and OSQ}

Mean score, standard deviations and Cronbach $\alpha$ values of SMB, SMVM and OSQ are given in table 2. Alpha values indicate that all scales and subscales are reliable.

Table 2. Means, standard deviations and Cronbach $\alpha$ values for SMBM, SMVM and OSQ

\begin{tabular}{lllll}
\hline Measures & Item & $M$ & $S D$ & Cronbach $\alpha$ \\
\hline SMBM & 14 & 2.78 & 0.43 & .91 \\
PF (physical fatigue) & 6 & 3.11 & 0.36 & .93 \\
CW (cognitive weariness) & 5 & 2.71 & 0.25 & .89 \\
EEx (emotional exhaustion) & 3 & 2.27 & 0.20 & .86 \\
SMVM & 12 & 5.16 & 0.22 & .88 \\
PHY (physical Strength) & 5 & 5.04 & 0.12 & .87 \\
CL (cognitive liveliness) & 3 & 5.25 & 0.06 & .84 \\
EE (emotional energy) & 4 & 5.23 & 0.34 & .86 \\
OSQ & 20 & 3.45 & 0.44 & .85 \\
\hline
\end{tabular}


Table 3 shows the results of t-test analysis of SMBM, SMVM and OSQ in terms of gender. Although female academicians have slightly higher scores for SMBM and its sub-dimensions, difference is not statistically significant. When SMVM is considered, male academicians' scores are slightly higher but with no significant difference. For OSQ scores, even though female academicians' scores were higher, difference was not big enough for a significant result.

Table 3. Comparison of scores (SMBM, SMVM, OSQ) by gender

\begin{tabular}{lllllcl}
\hline & $t$ & $d f$ & $p$ & \multicolumn{2}{c}{ Mean Difference SE Difference Cohen's $d$} \\
\hline SMBM & 0.929 & 40.00 & 0.358 & 0.247 & 0.266 & 0.290 \\
PF & 0.866 & 40.00 & 0.391 & 0.285 & 0.329 & 0.270 \\
CW & 0.366 & 40.00 & 0.716 & 0.125 & 0.341 & 0.114 \\
EEx & 1.047 & 40.00 & 0.302 & 0.375 & 0.358 & 0.326 \\
SMVM & -0.811 & 40.00 & 0.422 & -0.188 & 0.231 & -0.253 \\
PHY & -1.013 & 40.00 & 0.317 & -0.244 & 0.241 & -0.316 \\
CL & -0.984 & 40.00 & 0.331 & -0.333 & 0.339 & -0.307 \\
EE & -0.021 & 40.00 & 0.983 & -0.007 & 0.327 & -0.007 \\
OSQ & 1.328 & 40.00 & 0.192 & 0.197 & 0.149 & 0.414 \\
\hline
\end{tabular}

Note. Negative $t$ values indicate that scores of males $(n=18)$ are higher than females $(n=24)$.

In table 4, comparison of scores by age is given. As it is seen in the table, none of the scores differentiate according to age. Table 4. Comparison of scores (SMBM, SMVM, OSQ) by age

\begin{tabular}{lllllcl}
\hline & $t$ & $d f$ & $p$ & \multicolumn{2}{c}{ Mean Difference SE Difference Cohen's $d$} \\
\hline SMBM & 0.321 & 40.00 & 0.750 & 0.085 & 0.266 & 0.099 \\
PF & 0.235 & 40.00 & 0.815 & 0.077 & 0.328 & 0.073 \\
CW & -0.030 & 40.00 & 0.977 & -0.010 & 0.339 & -0.009 \\
EEx & 0.729 & 40.00 & 0.470 & 0.261 & 0.358 & 0.225 \\
SMVM & -0.671 & 40.00 & 0.506 & -0.154 & 0.230 & -0.207 \\
PHY & -0.649 & 40.00 & 0.520 & -0.156 & 0.241 & -0.201 \\
CL & -0.541 & 40.00 & 0.591 & -0.183 & 0.339 & -0.167 \\
EE & -0.401 & 40.00 & 0.691 & -0.130 & 0.323 & -0.124 \\
OSQ & -0.913 & 40.00 & 0.367 & -0.136 & 0.149 & -0.282 \\
\hline
\end{tabular}

Note. Negative $t$ values indicate that scores of participants above median age $(n=20)$ are higher than rest $(n=22)$.

As it is seen in table 5, none of SMBM, SMVM or OSQ scores differentiate in terms of years of service. However, difference between physical fatigue scores of senior and novice academicians is worth mentioning. Although it is not significant ( $p=0.076$ ) seniors PF scores are higher than novices (Mean Difference $=0.575$ ).

Table 5. Comparison of scores (SMBM, SMVM, OSQ) by years of service

\begin{tabular}{lllllll}
\hline & $t$ & $d f$ & $p$ & \multicolumn{2}{c}{ Mean Difference SE Difference Cohen's $d$} \\
\hline SMBM & -1.051 & 40.00 & 0.300 & -0.276 & 0.263 & -0.325 \\
PF & -1.820 & 40.00 & 0.076 & -0.575 & 0.316 & -0.562 \\
CW & -0.425 & 40.00 & 0.673 & -0.144 & 0.338 & -0.131 \\
EEx & 0.282 & 40.00 & 0.779 & 0.102 & 0.360 & 0.087 \\
SMVM & -0.883 & 40.00 & 0.383 & -0.202 & 0.229 & -0.273 \\
PHY & -0.173 & 40.00 & 0.864 & -0.042 & 0.242 & -0.053 \\
CL & -0.732 & 40.00 & 0.469 & -0.247 & 0.338 & -0.226 \\
EE & -1.156 & 40.00 & 0.255 & -0.368 & 0.318 & -0.357 \\
OSQ & -0.750 & 40.00 & 0.457 & -0.112 & 0.149 & -0.232 \\
\hline
\end{tabular}

Note. Negative $t$ values indicate that scores of participants above median years of service $(n=20)$ are higher than rest $(n$ =22).

As table 6 shows, the only score differentiate according to the number of doctor visits in a year is OSQ. Academicians with lower number of doctor visits have higher occupational satisfaction, $t(40)=2.136, p=.039$, with a medium-sized effect, $d=0.662$. 
Table 6. Comparison of scores (SMBM, SMVM, OSQ) by the number of doctor visits in a year

\begin{tabular}{lllllcl}
\hline & $t$ & $d f$ & $p$ & \multicolumn{3}{c}{ Mean Difference SE Difference Cohen's $d$} \\
\hline SMBM & 0.660 & 40.00 & 0.513 & 0.175 & 0.266 & 0.205 \\
PF & 0.156 & 40.00 & 0.877 & 0.051 & 0.330 & 0.048 \\
CW & 1.147 & 40.00 & 0.258 & 0.384 & 0.335 & 0.355 \\
EEx & 0.211 & 40.00 & 0.834 & 0.076 & 0.361 & 0.066 \\
SMVM & 0.511 & 40.00 & 0.612 & 0.118 & 0.231 & 0.158 \\
PHY & 0.605 & 40.00 & 0.548 & 0.146 & 0.242 & 0.188 \\
CL & -0.143 & 40.00 & 0.887 & -0.049 & 0.341 & -0.044 \\
EE & 0.643 & 40.00 & 0.524 & 0.208 & 0.323 & 0.199 \\
OSQ & 2.136 & 40.00 & $\mathbf{0 . 0 3 9}$ & 0.305 & 0.143 & 0.662 \\
\hline
\end{tabular}

Note. Negative $t$ values indicate that scores of participants with above median doctor visit number $(n=19)$ are higher than rest $(n=23)$.

Table 7 shows academicians who feel more healthy have lower SMBM scores, $t(40)=2.448, p=.019$, with a medium-sized effect, $d=0.769$. Similarly those academicians have lower PF scores, $t(40)=2.483, p=.017$, with a medium-sized effect, $d=0.781$. Although those academicians' CW scores were lower as well, this difference was not significant. The OSQ scores differentiate in favour of academicians with higher health status, $t(40)=-2.476, p=.018$, with a medium-sized effect, $d=-0.778$.

Table 7. Comparison of scores (SMBM, SMVM, OSQ) by health status

\begin{tabular}{lllllcl}
\hline & $t$ & $d f$ & $p$ & \multicolumn{2}{c}{ Mean Difference SE Difference Cohen's $d$} \\
\hline SMBM & 2.448 & 40.00 & $\mathbf{0 . 0 1 9}$ & 0.618 & 0.253 & 0.769 \\
PF & 2.483 & 40.00 & $\mathbf{0 . 0 1 7}$ & 0.773 & 0.311 & 0.781 \\
CW & 1.221 & 40.00 & 0.229 & 0.413 & 0.338 & 0.384 \\
EEx & 1.852 & 40.00 & 0.071 & 0.651 & 0.351 & 0.582 \\
SMVM & -0.493 & 40.00 & 0.625 & -0.115 & 0.234 & -0.155 \\
PHY & -1.549 & 40.00 & 0.129 & -0.371 & 0.239 & -0.487 \\
CL & 0.377 & 40.00 & 0.708 & 0.130 & 0.345 & 0.119 \\
EE & 0.059 & 40.00 & 0.953 & 0.019 & 0.329 & 0.019 \\
OSQ & -2.476 & 40.00 & $\mathbf{0 . 0 1 8}$ & -0.353 & 0.142 & -0.778 \\
\hline
\end{tabular}

Note. Negative $t$ values indicate that scores of participants with above median health status $(n=17)$ are higher than rest $(n=25)$.

As table 8 indicates, burnout and vigor have negative correlation. Occupational satisfaction has negatively correlated with emotional exhaustion, which is a sub-dimension of burnout but positively correlated with physical strength, which is a sub-dimension of vigor.

Table 8. Pearson Correlations

\begin{tabular}{|c|c|c|c|c|c|c|c|c|}
\hline & SMBM & $\mathrm{PF}$ & $\mathrm{CW}$ & EEx & SMVM PHY & $\mathrm{CL}$ & $\mathrm{EE}$ & OSQ \\
\hline \multicolumn{9}{|c|}{ SMBM - } \\
\hline $\mathrm{PF}$ & $0.832^{* * * *}$ & & & & & & & \\
\hline $\mathrm{CW}$ & $0.822^{* * *}$ & $0.469^{* * *}$ & - & & & & & \\
\hline EEx & $0.642^{* * *}$ & $0.311^{*}$ & $0.413^{* * *}$ & - & & & & \\
\hline SMVM & $-0.415^{* *}$ & $-0.308^{*}$ & -0.277 & $-0.435^{* *}$ & - & & & \\
\hline PHY & $-0.512^{* * * *}$ & $-0.546^{* * * *}$ & $-0.355^{*}$ & -0.214 & $0.816^{* * * *}-$ & & & \\
\hline $\mathrm{CL}$ & $-0.308^{*}$ & -0.188 & -0.262 & $-0.310^{*}$ & $0.821^{* * * *} 0.651^{* * * *}$ & - & & \\
\hline $\mathrm{EE}$ & -0.166 & -0.000 & -0.055 & $-0.486^{* *}$ & $0.731^{* * * *} 0.299$ & $0.360^{*}$ & - & \\
\hline OSQ & -0.266 & -0.213 & -0.140 & $-0.311^{*}$ & $0.2580 .309^{*}$ & 0.170 & 0.130 & - \\
\hline
\end{tabular}

When mean scores are accepted as cut-off point, 19 academicians (45.2\%) had burnout. 12 (63.2\%) of those who had burnout were female. Twenty academicians (47.6\%) were vigorous and $10(50 \%)$ of those were female. Totally 26 academicians' $(61.9 \%)$ OSQ scores were higher than the average score. Of those who had higher scores $15(58 \%)$ were female.

\section{Conclusion and Discussion}

This study has set out to find out the academicians' level of burnout, vigor and occupational satisfaction, working at a Fine Arts Education Department. The study contributes to our understanding of key points which may affect the efficacy of academicians' work force and wellbeing at their jobs.

The results related to burnout levels of the academicians suggest that even if the female academicians' level of burnout 
is slightly higher than male academicians', the sample size is not sufficient to detect significant difference. When literature is reviewed, there are some studies who find significantly higher female scores and some studies are unable to detect any difference in terms of gender. For example; Derinbay (2012) states that emotional exhaustion (a sub-dimension of burnout) is higher among female academicians, but there is no significant difference in other sub-dimensions (depersonalization and personal success levels) or total burnout scores. This finding is consistent with that of Cavusoglu et al. (2015) who state female academicians have significantly higher level of burnout when compared to male academicians.

The results of vigor levels of the academicians show that male academicians' level of vigor is slightly higher than female academicians' in all of the sub-dimensions and total of SMVM. However, the differences are not significant. Similarly, in the study by Bayram et al. (2010) male academicians are found to be more vigorous than females significantly, except EE sub-dimension of SMVM.

When it comes to occupational satisfaction questionnaire, female academicians seem to have higher occupational satisfaction, although there is no statistically significant difference between scores. This result is not consistent with data obtained in Derinbay (2012)'s study in which she states that occupational satisfaction is higher among male academicians. However, in another study by Bilge, Akman and Kelecioglu (2007), they find no significant relation between the variables of occupational satisfaction and gender.

Kucuksuleymanoglu (2007), whose study is carried out in the Department of Education at Uludag University, states that academicians age between 21 and 30 have higher level of burnout. However, in any of the dimensions of this current study, it turns out that age variable does not have any impact. Also, we find that the level of physical fatigue seems to increase according to the years of service, even though it is not significant.

This study also takes health status of the academicians into consideration. The results reveal that the academicians who go to the doctor less frequently have higher occupational satisfaction. Moreover, the academicians who are facing health issues have higher level of burnout. The results confirm that health status significantly affects one sub-dimension of burnout, which is physical fatigue. Although the result is not significant, academicians who have declared that their health status is good have lower level of emotional exhaustion, whereas cognitive weariness is not affected. However, there was no significant difference among academicians' vigor scores in terms of health status.

From the point of correlational relations, it is seen that burnout and vigor have negative correlation. Occupational satisfaction has negatively correlated with emotional exhaustion, which is a sub-dimension of burnout but positively correlated with physical strength, which is a sub-dimension of vigor. Derinbay (2012) also detects negative correlation between occupational satisfaction and burnout.

Taken together, the current data highlight the importance of replicating this type of studies frequently as they are essential for an institution to operate at full capacity. Administrations should be determined to carry on observing the problems and support academicians to create constructive atmosphere.

\section{Suggestions}

This work contributes to existing knowledge of academicians' level of burnout, vigor and occupational satisfaction by adding the specific case of Fine Arts Education Department at Uludag University. Since educators working in all levels of education are vital for the education system to work efficiently and effectively, their burnout levels should regularly be determined and necessary improvements should be made to create fruitful job atmosphere for them. Educators should feel satisfied with their jobs in order to be creative and productive. If necessary steps should not be taken and educators are left alone, nothing much can be expected than failure in all steps in education system. Thus, these kinds of studies must be done repetitively.

\section{References}

Bayram, N., Gursakal, S., \& Bilgel, N. (2010). Burnout, vigor and job satisfaction among academic staff. European Journal of Social Sciences, 17(1), 41-53.

Bilge, F., Akman, Y., \& Kelecioglu, H. (2007). Examining the job satisfaction of academics. Hacettepe University Journal of Education, 32, 32-41.

Bilgel, N., Bayram, N., Ozdemir, H., Dogan, F., \& Ekin, D. (2012). Work engagement, burnout and vigor among a group of medical residents in Turkey. British Journal of Education, Society \& Behavioural Science, 2(3), 220-238. Retrieved from https://doi.org/10.9734/BJESBS/2012/1496

Cavusoglu, G., Unver, S., Dogan, E., Islamoglu, I., \& Ozdemir, S. (2015). Comparison of the burnout levels of academics in the faculty of sports sciences and other faculties. Spor Yonetimi ve Bilgi Teknolojileri Dergisi, 10(1), $4-13$. 
Cooper, C. (2016). The secret recipe for high-performing schools? Heads who listen to teachers. The Guardian. Retrieved https://www.theguardian.com/teacher-network/2016/apr/21/high-performing-schools-heads-listen-teachers-staff-w ellbeing

Derinbay, D. (2012). Examining the job satisfaction and professional burnout of academics (samples of Pamukkale University). E-Journal of New World Sciences Academy, 6(3), 910-929.

Evans, S., Huxley, P., Gately, C., Webber, M., Mears, A., Pajak, A. H., ... Katona, C. (2006). Mental health, burnout and job satisfaction among mental health social workers in England and Wales. The British Journal of Psychiatry, 188(1), 75-80. https://doi.org/10.1192/bjp.188.1.75

Filiz, Z. (2014). An analysis of the levels of job satisfaction and burnout of teachers. Journal of Management Economics and Business, 10(23), 157-172.

Fraenkel, J. R., Wallen, N. E., \& Hyun, H. H. (2012). How to design and evaluate research in education (8th ed.). NY: McGraw-Hill.

Freudenberger, H. J. (1974). Staff burn-out. Journal of Social Issues, 30(1), 159-165. https://doi.org/10.1111/j.1540-4560.1974.tb00706.x

Gursel, M., Sunbul, A. M., \& Sari, H. (2002). An analysis of burnout and job satisfaction between Turkish headteachers and teachers. European Journal of Psychology of Education, 17(1), 35-45. https://doi.org/10.1007/BF03173203

Karabiyik, L., Eker, M., \& Anbar, A. (2008). Determining the factors that affect burnout among academicians. Ankara University Journal of Social Sciences, 63(2), 91-115. Retrieved from http://dergipark.ulakbim.gov.tr/ausbf/article/download/5000053395/5000050712

Kilic, S., Tanrikulu, T., \& Ugur, H. (2013). Job satisfaction and social comparison levels of teachers working for state schools. International Journal of Human Sciences, 10(1), 760-779.

Koustelios, A., \& Tsigilis, N. (2005). The relationship between burnout and job satisfaction among physical education teachers: a multivariate approach. European Physical Education Review, 11(2), 189-203. https://doi.org/10.1177/1356336X05052896

Kucuksuleymanoglu, R. (2007). Burn out syndrome levels of education faculty academic staff. Eurasian Journal of Educational Research, 28, 101-112.

Maslach, C., Jackson, S. E., \& Leiter, M. P. (1996). Maslach burnout inventory manual. Palo Alto (3rd ed.). CA: Consulting Psychologists Press.

Maslach, C., Schaufeli, W. B., \& Leiter, M. P. (2001). Job burnout. Annual Review of Psychology, 52(1), 397-422. https://doi.org/10.1146/annurev.psych.52.1.397

Ogresta, J., Rusac, S., \& Zorec, L. (2008). Relation between burnout syndrome and job satisfaction among mental health workers. Croatian Medical Journal, 49(3), 364-374. Retrieved from https://doi.org/10.3325/cmj.2008.3.364

Otacioglu, S. G. (2008). Burnout syndrome among music teachers and triggering factors. Inonu University Journal of the Faculty of Education, 9(15), 103-116.

Ozyurt, A., Hayran, O., \& Sur, H. (2006). Predictors of burnout and job satisfaction among Turkish physicians. QJM: Monthly Journal of the Association of Physicians, 99(3), 161-169. https://doi.org/10.1093/qjmed/hc1019

Schaufeli, W. B., \& Bakker, A. B. (2004). Job demands, job resources, and their relationship with burnout and engagement: a multi-sample study. Journal of Organizational Behavior, 25(3), 293-315.

https://doi.org/10.1002/job.248

Schaufeli, W. B., \& Enzmann, D. (1998). The burnout companion to study and practice: A critical analysis. Taylor \& Francis. Retrieved from https://books.google.co.uk/books?id=cL88XbNVv8QC\&lpg=PR9\&ots=keNjsTIAuL\&dq=The\%20burnout\%20co mpanion $\% 20$ to $\% 20$ study $\% 20$ and $\% 20$ practice: $\% 20 \mathrm{~A} \% 20$ critical\%20analysis\&lr\&pg=PP1\#v=onepage \&q=The\%2 Oburnout $\% 20$ companion $\% 20$ to $\% 20$ study $\% 20$ and $\% 20$ practice $\% 3 \mathrm{~A} \% 20 \mathrm{~A} \% 20 \mathrm{critical} \% 20$ analysis\&f $=$ false

Schaufeli, W. B., Salanova, M., González-romá, V., \& Bakker, A. B. (2002). The Measurement of Engagement and Burnout: A Two Sample Confirmatory Factor Analytic Approach. Journal of Happiness Studies, 3(1), 71-92. https://doi.org/10.1023/A:1015630930326

Shirom, A., \& Melamed, S. (2005). Shirom-Melamed burnout and vigor measures. Retrieved February 10, 2018, from http://www.shirom.org/arie/index.html\# 


\section{Appendix}

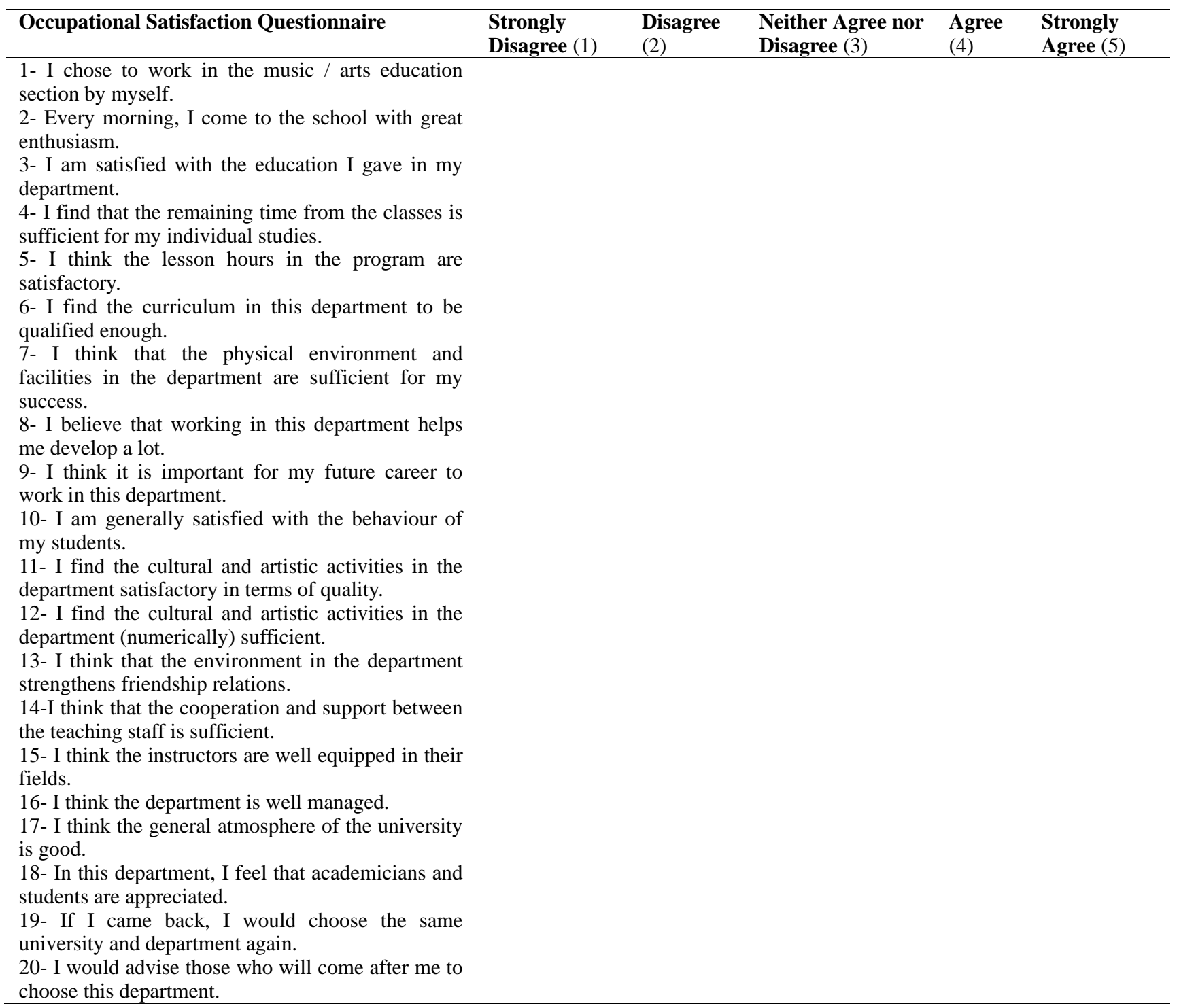

\section{Copyrights}

Copyright for this article is retained by the author(s), with first publication rights granted to the journal.

This is an open-access article distributed under the terms and conditions of the Creative Commons Attribution license which permits unrestricted use, distribution, and reproduction in any medium, provided the original work is properly cited. 